\title{
Quantitative analysis of annealing-induced instabilities of photo-leakage current and negative-bias-illumination-stress in a-InGaZnO thin-film transistors
}

\author{
Dapeng Wang ${ }^{* 1}$ and Mamoru Furuta ${ }^{* 2,3}$
}

\author{
Full Research Paper \\ Address: \\ ${ }^{1}$ Key Laboratory of Applied Surface and Colloid Chemistry, Ministry of \\ Education; Shaanxi Key Laboratory for AdvancedEnergy Devices; \\ Shaanxi Engineering Lab for Advanced Energy Technology, School of \\ Materials Science and Engineering, Shaanxi Normal University, Xi'an \\ 710119, China, ${ }^{2}$ School of Environmental Science and Engineering, \\ Kochi University of Technology, Kami, Kochi 782-8502, Japan and \\ ${ }^{3}$ Center for Nanotechnology in Research Institute, Kochi University of \\ Technology, Kami, Kochi 782-8502, Japan

\section{Email:} \\ Dapeng Wang* - dpwang@snnu.edu.cn; Mamoru Furuta* - \\ furuta.mamoru@kochi-tech.ac.jp \\ * Corresponding author \\ Keywords: \\ metal oxide; photo-induced instabilities; photon energy; thermal \\ annealing; thin-film transistor (TFT) device

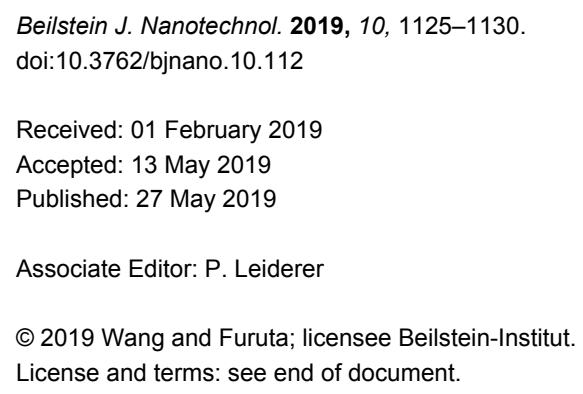

Open Access

\begin{abstract}
This study examines the effect of the annealing temperature on the initial electrical characteristics and photo-induced instabilities of amorphous indium gallium zinc oxide (a-IGZO) thin-film transistors (TFTs). The extracted electrical parameters from transfer curves suggest that a low-temperature treatment maintains a high density of defects in the IGZO bulk, whereas high-temperature annealing causes a quality degradation of the adjacent interfaces. Light of short wavelengths below $460 \mathrm{~nm}$ induces defect generation in the forward measurement and the leakage current increases in the reverse measurement, especially for the low-temperatureannealed device. The hysteresis after negative-bias-illumination-stress (NBIS) is quantitatively investigated by using the doublescan mode and a positive gate pulse. Despite the abnormal transfer properties in the low-temperature-treated device, the excited holes are identically trapped at the front interface irrespective of treatment temperature. NBIS-induced critical instability occurs in the high-temperature-annealed TFT.
\end{abstract}

\section{Introduction}

The rapid process of industrialization and commercialization has accelerated the development of consumption electronics and micromachining technology. One of the most successful modern-day microelectronic products are metal-oxide thin-film transistors (TFTs) that guarantee large-scale integrated circuits for applications in transparent and flexible flat-panel displays (FPDs). Amorphous InGaZnO (a-IGZO), an outstanding active channel material, is generally adopted in TFTs because of 
its high electron mobility, great environmental/thermal stability, and preparation versatility. The enhanced mobility of a-IGZO originates from the fact that the electrical conduction is insensitive to disorder because the conduction band edge depends on the In s-orbital [1]. However, there is a high density of oxygen-containing trap states (ca. $10^{20} \mathrm{~cm}^{-3}$ ) in the region above the valence band [2], which is sensitive to irradiation with light [3].

The typical staggered/coplanar TFT devices comprise several functional layers and their respective contact interfaces. Generally, the individual layer fabrication process follows a number of thin film deposition and photolithographic patterning steps. During the film growth through plasma-enhanced chemical vapor deposition or sputtering techniques, the sample suffers from the plasma bombardment in the chamber. In addition, the deposited films are inevitably irradiated by UV light in the process of patterning, inducing the increase in the concentration of oxygen-related defects, which further degrade the initial electrical properties and long-term stability. Therefore, many researches are devoted to reducing the density of defects during the fabrication, including the optimization of oxygen partial pressure [4], the increase of fabrication temperature, and the reduction of deposition power. In addition, post-annealing is recognized as an essential method to enhance the quality of the channel layer as well as its adjacent interfaces [5].

Although the initial performance of the TFTs can be improved using a passivation layer and suitable post-annealing, the devices in FPDs always undergo a negative gate bias and are exposed to the backlight for non-emissive displays. The degradation of oxide-based TFTs under this kind of negative-biasillumination-stress (NBIS) is a key issue that has been investigated over the last decade [6,7]. Despite all efforts to unveil the mechanisms of NBIS, such as first-principles calculations and experimental researches, the NBIS-triggered degradations in devices annealed at different temperatures are still not fully understood. Furthermore, the photo-leakage current and NBISderived instabilities of devices annealed at different temperatures have not been quantitatively traced so far.

In this work, we investigate the impact of annealing temperature on the initial electrical characteristics and photo-induced instabilities in a-IGZO TFTs. The obtained electrical parameters imply that low- and high-temperature annealing leverage the generation of defects in the active layer as well as its adjacent interfaces. The incident photon energy $(>2.7 \mathrm{eV})$ excites the defect generation and the leakage current increases in the devices regardless of heating temperature. By means of the double-scan mode and a positive gate pulse, the NBIS-caused hysteresis of the TFTs annealed at different temperatures is quantitatively monitored to trace trapped holes, generated donor-like states, and trapped electrons.

\section{Experimental}

The bottom-gate top-contact a-IGZO TFTs are fabricated in a routine procedure [8]. The channel layer thickness is $45 \mathrm{~nm}$, and then the TFTs are annealed at 300,350 , and $400{ }^{\circ} \mathrm{C}$ in $\mathrm{N}_{2}$ atmosphere for $1 \mathrm{~h}$. The TFTs are designed with fixed width $(W)$ of $50 \mu \mathrm{m}$ and length $(L)$ of $20 \mu \mathrm{m}$. The current-voltage $(I-V)$ curves are measured using an Agilent $4156 \mathrm{C}$ semiconductor parameter analyzer at room temperature.

For the photo-leakage current measurements, monochromatic light with wavelengths $\lambda$ of $400-530 \mathrm{~nm}$ is used. The details of the NBIS measurement (gate voltage $V_{\mathrm{GS}}=-20 \mathrm{~V} ; \lambda=460 \mathrm{~nm}$ ) in double-scan mode are described in [8]. The duration of the NBIS measurements is $10^{4} \mathrm{~s}$.

\section{Results and Discussion}

Figure 1 displays the double-scan transfer characteristics $\left(V_{\mathrm{DS}}=10.1 \mathrm{~V}\right)$ of the TFTs annealed at different temperatures. The extracted electrical parameters as functions of the treatment temperature are listed in Table 1 . The field-effect mobility

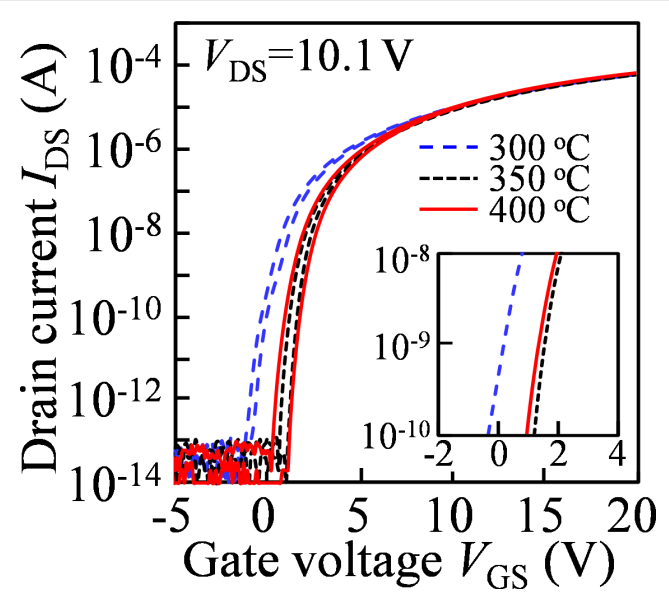

Figure 1: Transfer characteristics of the devices annealed at different temperatures.

Table 1: The electrical parameters of a-IGZO TFTs annealed at different temperatures.

annealing temperature $\left({ }^{\circ} \mathrm{C}\right)$

$300 \quad 350 \quad 400$

\begin{tabular}{llll}
\hline$\mu_{\text {sat }}\left(\mathrm{cm}^{2} \cdot \mathrm{V}^{-1} \cdot \mathrm{s}^{-1}\right)$ & 14.75 & 14.67 & 14.79 \\
$V_{\text {on }}$ at $I_{\mathrm{DS}}=1 \mathrm{nA}(\mathrm{V})$ & 0.17 & 1.53 & 1.33 \\
hysteresis $\Delta V_{\mathrm{H}}(\mathrm{V})$ & 0.38 & 0.37 & 0.77 \\
subthreshold swing $(\mathrm{mV} / \mathrm{dec})$. & 369 & 250 & 292 \\
total trap density $\left(10^{11} \mathrm{~cm}^{-2}\right)$ & 7.67 & 4.72 & 5.76
\end{tabular}


$\left(\mu_{\mathrm{sat}}\right)$ is directly proportional to the drain-to-source current $\left(I_{\mathrm{DS}}\right)$ [9]. The mobility results demonstrate that the post-annealing temperature scarcely changes the $I_{\mathrm{DS}}$ values. The turn-on voltage $V_{\text {on }}$ (at $I_{\mathrm{DS}}=1 \mathrm{nA}$ ) of $0.17 \mathrm{~V}$ for the device treated at $300{ }^{\circ} \mathrm{C}$ changes to 1.53 and $1.33 \mathrm{~V}$ for the TFTs annealed at $350^{\circ}$ and $400{ }^{\circ} \mathrm{C}$, respectively. The shift of $V_{\text {on }}$ in the positive direction suggests that a proper treatment temperature is beneficial for the formation of the metal-oxide atomic framework, improving the channel layer quality. The notorious $I-V$ hysteresis is regarded as an indicator for the quality of the GI/semiconductor interface. The values of the hysteresis, i.e., the difference of $V_{\mathrm{GS}}$ at $I_{\mathrm{DS}}=1 \mathrm{pA}$ scanned in the forward and reverse directions, are $0.38,0.37$, and $0.77 \mathrm{~V}$, for the devices annealed at 300,350 , and $400{ }^{\circ} \mathrm{C}$, respectively. The large hysteresis implies that the high-temperature treatment causes the deterioration of the front interface. Furthermore, the subthreshold swing ( $\left.\mathrm{SS}=\mathrm{d} V_{\mathrm{GS}} / \mathrm{dlog}_{10}\left(I_{\mathrm{DS}}\right)\right)$ is calculated to be 369,250 , and $292 \mathrm{mV} /$ dec. for the TFTs treated at 300,350 , and $400{ }^{\circ} \mathrm{C}$, respectively. Note that the SS is a measure for the total density of trap states $\left(N_{\mathrm{t}}\right)$ in the semiconductor bulk and the front interface of a TFT device. On the basis of the functional relationship between of SS and $N_{\mathrm{t}}$ [10], an $N_{\mathrm{t}}$ value of $7.67 \times 10^{11} \mathrm{~cm}^{-2} \cdot \mathrm{eV}^{-1}$ is obtained for the TFT treated at $300{ }^{\circ} \mathrm{C}$. From the comprehensive analysis of the hysteresis and SS results, we concluded that the low-temperature-annealed TFT exhibits the high density of defects in the IGZO bulk, whereas the high-temperature-treated device has severe defect states at the GI/IGZO interface. On the basis of our previous publication [11], the results suggest that the appropriate annealing temperature is helpful to suppress the defects and strengthen the quality of a-IGZO. However, the high temperature will induce a rupture of weak metal-oxygen bonds, introducing more defect sites.

Figure 2a-f shows the transfer curves of the devices measured in double-scan mode without and with monochromatic light illumination. In the forward measurement, when the incident light wavelength is shorter than $460 \mathrm{~nm}$, a SS degradation is distinctly observed regardless of the annealing temperature. The extent of deterioration is lower for increasing treatment temperatures, which further proves that the TFT annealed at low temperature maintains the high concentration of defects in the channel layer. The SS degradation is effectively depressed in the reverse measurement even under higher-energy light irradiation, demonstrating that the generated ionized oxygen vacancies $\left(\mathrm{V}_{\mathrm{O}}{ }^{+} / \mathrm{V}_{\mathrm{O}}{ }^{2+}\right)$ are neutralized by capturing electrons [8]. However, SS degradation still can be observed in the $300{ }^{\circ} \mathrm{C}$-annealed TFT under the irradiation with short-wavelength light (below $430 \mathrm{~nm}$ ), indicating that light-induced defect states cannot be completely stabilized. In addition, a photoleakage current starts to appear when the wavelength of the incident light is $460 \mathrm{~nm}$. The current gradually increases with a decrease in light wavelength and annealing temperature. The influence of the incident light energy on the photo-leakage current of the devices annealed at different temperatures is displayed in Figure 2g. From using the double-scan mode, it can be concluded that the low-temperature-treated IGZO contains intrinsic defect states around the Fermi level $\left(E_{\mathrm{F}}\right)$ at $V_{\mathrm{on}}$, which
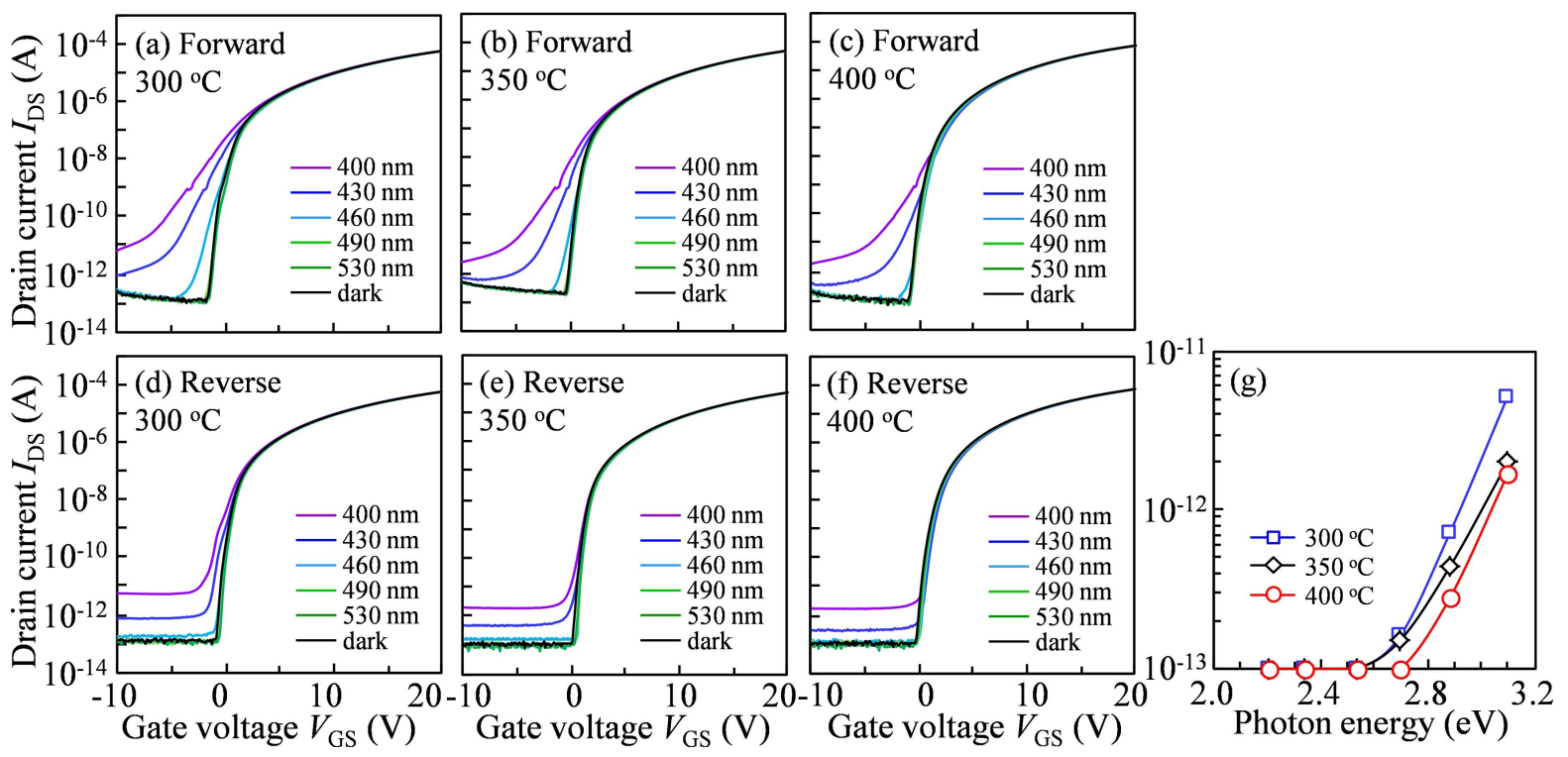

Figure 2: Transfer curves scanned in the forward direction for a-IGZO TFTs annealed at (a) $300{ }^{\circ} \mathrm{C}$, (b) $350{ }^{\circ} \mathrm{C}$, and (c) $400{ }^{\circ} \mathrm{C}$ and scanned in the reverse direction for the devices treated at (d) $300{ }^{\circ} \mathrm{C}$, (e) $350{ }^{\circ} \mathrm{C}$, and (f) $400{ }^{\circ} \mathrm{C}$ under monochromatic light irradiation; (g) photo-leakage current $\left(V_{G S}=-10 \mathrm{~V}\right.$ and $\left.V_{D S}=10 \mathrm{~V}\right)$ of a-IGZO TFTs in the reverse measurement as a function of photon energy of the incident light. 
are added to the light-excited defect states at the same level. It is also implied that the electrical properties, including SS and offcurrent, directly depend on the annealing temperature regardless whether or not there is light irradiation.

The devices annealed at different temperatures were then exposed to a NBIS measurement over a period of $10^{4} \mathrm{~s}$ (Figure 3). In the forward measurement (Figure 3a-c), the initial transfer curve of the TFT treated at $300{ }^{\circ} \mathrm{C}$ exhibits a serious SS deterioration. The extent of SS degradation is gradually aggravated along with on-current reduction with the increasing stress duration. The photo-induced current results indicate that the incident light with the wavelength of $460 \mathrm{~nm}$ (ca. $2.7 \mathrm{eV}$ ) causes a severe SS distortion irrespective of the treatment temperature. Therefore, new defect states are generated and accumulated near $E_{\mathrm{F}}$ at $V_{\mathrm{on}}$, which originate from the high density of $\mathrm{V}_{\mathrm{O}}$ near the valence band $\left(E_{\mathrm{V}}\right)$. When the annealing temperature increases to $350{ }^{\circ} \mathrm{C}$, the transfer curves exhibit outstanding initial electrical properties. However, a positive shift accompanied by the appearance of a hump and SS degradation occurs and gradually increases with increasing stress duration. The results demonstrate that the high concentration of $\mathrm{V}_{\mathrm{O}}$ defects near $E_{\mathrm{V}}$ is excited to $\mathrm{V}_{\mathrm{O}}{ }^{+} / \mathrm{V}_{\mathrm{O}}{ }^{2+}$, located below the bottom of the conduction band [12]. Meanwhile, the photo-excited electron-hole pairs from the $E_{\mathrm{V}}$ are separated because of a vertical electric field $\left(V_{\mathrm{GS}}=-20 \mathrm{~V}\right)$. Consequently, the transfer curves shifted in the positive direction are attributed to generated holes trapped in the GI/IGZO interface. These phenomena are stronger when the heating temperature is further increased to $400{ }^{\circ} \mathrm{C}$, suggesting that the more $\mathrm{V}_{\mathrm{O}}$ defects exist near the $E_{\mathrm{V}}$ region. In the reverse measurement (Figure 3d-f), the initial transfer curve in the $300{ }^{\circ} \mathrm{C}$-treated TFT still shows an acute SS distortion. The tendency of SS degradation is slightly strengthened, and there is a slightly positive shift of $2.51 \mathrm{~V}$ when the stress duration exceeds $1000 \mathrm{~s}$. These results imply that the generated defect states cannot be completely stabilized even through scanning with high voltage. There is no hump observed in the forward scanning measurement of the $350{ }^{\circ} \mathrm{C}$-annealed device, proving that light-induced defect states during the stress are stabilized when the measurement voltage is scanned from 20 to $-10 \mathrm{~V}$. Meanwhile, the transfer curves shift without SS distortion. In the case of the device annealed at $400{ }^{\circ} \mathrm{C}$, the transfer curves further shift to $10.29 \mathrm{~V}$ with a stable SS (Figure $3 \mathrm{~g}$ ) that is larger than that in the case of annealing at $350{ }^{\circ} \mathrm{C}(7.20 \mathrm{~V})$.

To quantitative evaluate the hysteresis caused by NBIS, a $10 \mathrm{~V}$ pulse with $1 \mathrm{~ms}$ width is exerted just after $10^{4} \mathrm{~s}$ stress duration and before the forward measurement. Figure $4 a-c$ shows the transfer curves of the annealed TFTs before and after stress without and with a pulse. After a stress duration of $10^{4} \mathrm{~s}$, the hysteresis values are $3.99,7.76$, and $10.55 \mathrm{~V}$ for the TFTs annealed at 300,350 , and $400{ }^{\circ} \mathrm{C}$, respectively (Figure $4 \mathrm{~d}$ ). In our previous publication [13], the additional positive gate pulse can neutralize the ionized $\mathrm{V}_{\mathrm{O}}^{+} / \mathrm{V}_{\mathrm{O}}{ }^{2+}$ states and release the trapped holes at the GI/IGZO interface, helping to smooth the hump induced by donor-like defect states. When the pulse is applied, the hysteresis values are $1.58,2.75$, and $2.66 \mathrm{~V}$ corre-
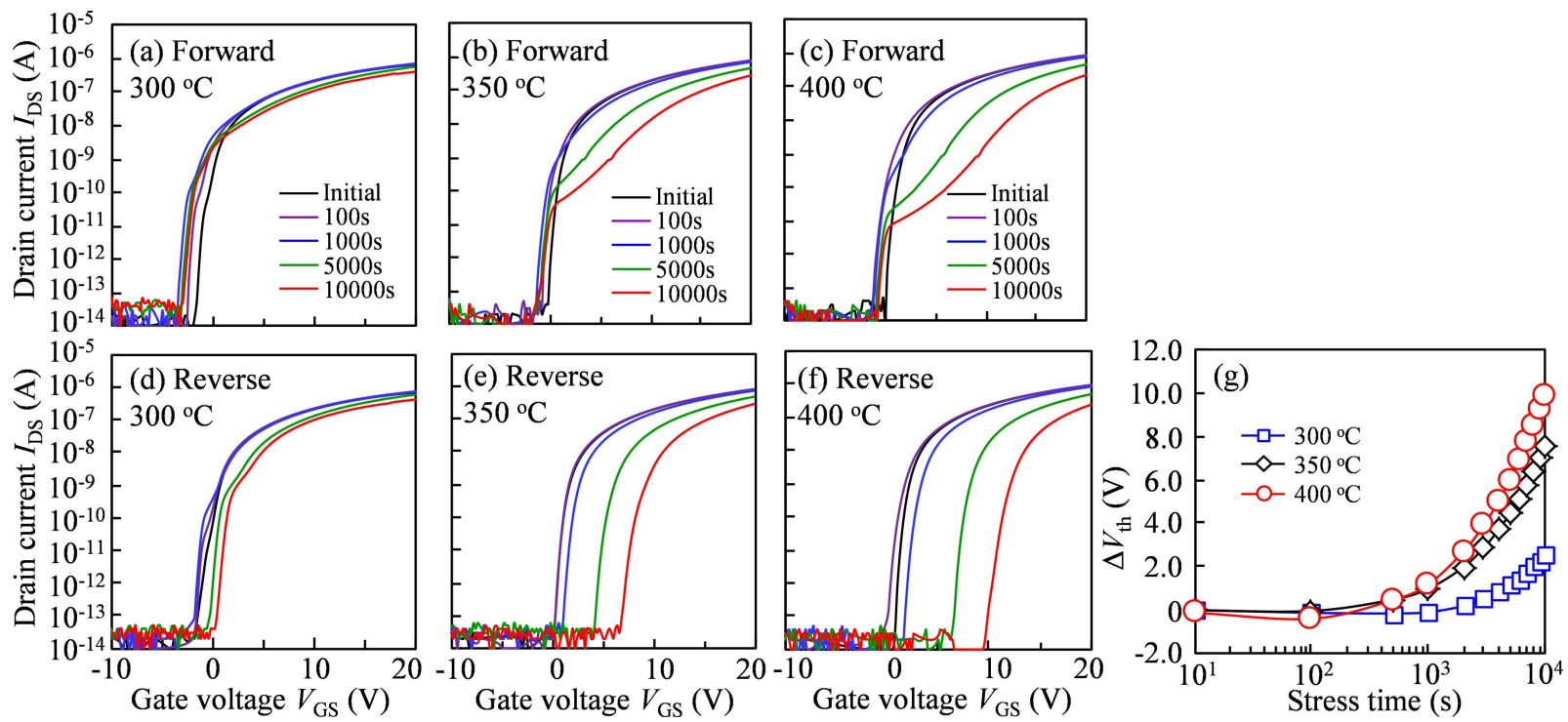

Figure 3: Transfer characteristics in the forward measurement for the devices annealed at (a) $300{ }^{\circ} \mathrm{C}$, (b) $350{ }^{\circ} \mathrm{C}$, and (c) $400{ }^{\circ} \mathrm{C}$, and in the reverse measurement for the devices annealed at (d) $300{ }^{\circ} \mathrm{C}$, (e) $350^{\circ} \mathrm{C}$, and (f) $400{ }^{\circ} \mathrm{C}$ as functions of stress duration under $-20 \mathrm{~V} V_{\mathrm{GS}} \mathrm{NBIS}$; (g) $V_{\text {th }}$ values of the transfer curves of the devices as a function of NBIS duration. 

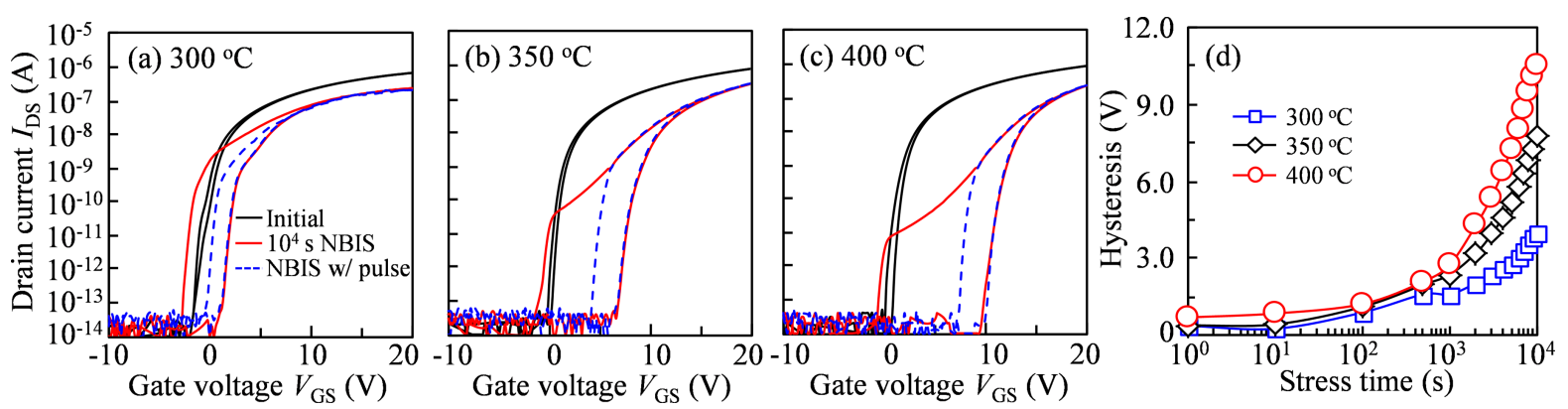

Figure 4: Figure 4 Transfer curves of IGZO TFTs annealed at (a) $300^{\circ} \mathrm{C}$, (b) $350{ }^{\circ} \mathrm{C}$, and (c) $400{ }^{\circ} \mathrm{C}$ before and after $10^{4} \mathrm{~s}$ NBIS without and with a $10 \mathrm{~V}$ gate pulse; (d) hysteresis of IGZO TFTs as a function of NBIS duration.

sponding for the devices treated at 300,350 , and $400{ }^{\circ} \mathrm{C}$, respectively. The total hysteresis induced by NBIS consists of defect-state generation in the bulk and hole trapping at the interface. The contribution of excited defect states to the hysteresis values is calculated to be $2.41,5.01$, and $7.89 \mathrm{~V}$ for devices annealed at 300,350 , and $400{ }^{\circ} \mathrm{C}$, respectively. In the TFT treated at $300{ }^{\circ} \mathrm{C}$, the incident light excites oxygen-containing defects to $\mathrm{V}_{\mathrm{O}}{ }^{+} / \mathrm{V}_{\mathrm{O}}{ }^{2+}$, which create new defect states in the IGZO. Meanwhile, electron-hole pairs are excited in the channel layer, which partly neutralize the defect states with free electrons, leaving holes near $E_{\mathrm{V}}$. Furthermore, the generated free holes and electrons are prone to be trapped because of more defect states existing in the IGZO layer (Figure 5a). Consequently, the transfer curve exhibits a severe SS degradation in the forward measurement, as well as a big SS distortion and a relatively small positive shift. In contrast, for the device annealed at $350{ }^{\circ} \mathrm{C}$, the created donor-like states in the forward measurement are completely stabilized in the reverse scanning, and photo-induced carriers are driven by the electric field and drift to the interfaces (Figure 5b). In virtue of the relative high quality of the IGZO bulk as well as its adjacent interfaces, the transfer curves show only a small hump and a small gap-shift without SS distortion in the forward and reverse scanning. When the TFT is annealed at $400{ }^{\circ} \mathrm{C}$, the attenuation of the electrical properties is equivalent to that of the $350{ }^{\circ} \mathrm{C}$ sample. However, due to the lesser quality of the IGZO layer and its adjacent interfaces, the transfer curves exhibit a big hump in the forward scan and a long-range shift in the reverse scan. Furthermore, when the double-scan mode and a positive gate pulse are combined, as schematically illustrated in Figure 5c, a higher number of defect states is excited in the semiconductor bulk and an identical amount of holes is trapped at the interfaces in comparison with the results in the TFT annealed at $350{ }^{\circ} \mathrm{C}$.

Based on the abovementioned results, it is suggested that the annealing process provides the possibility to regulate the qualities of the channel layer as well as its adjacent interfaces, thereby controlling the initial electrical characteristics and bias-
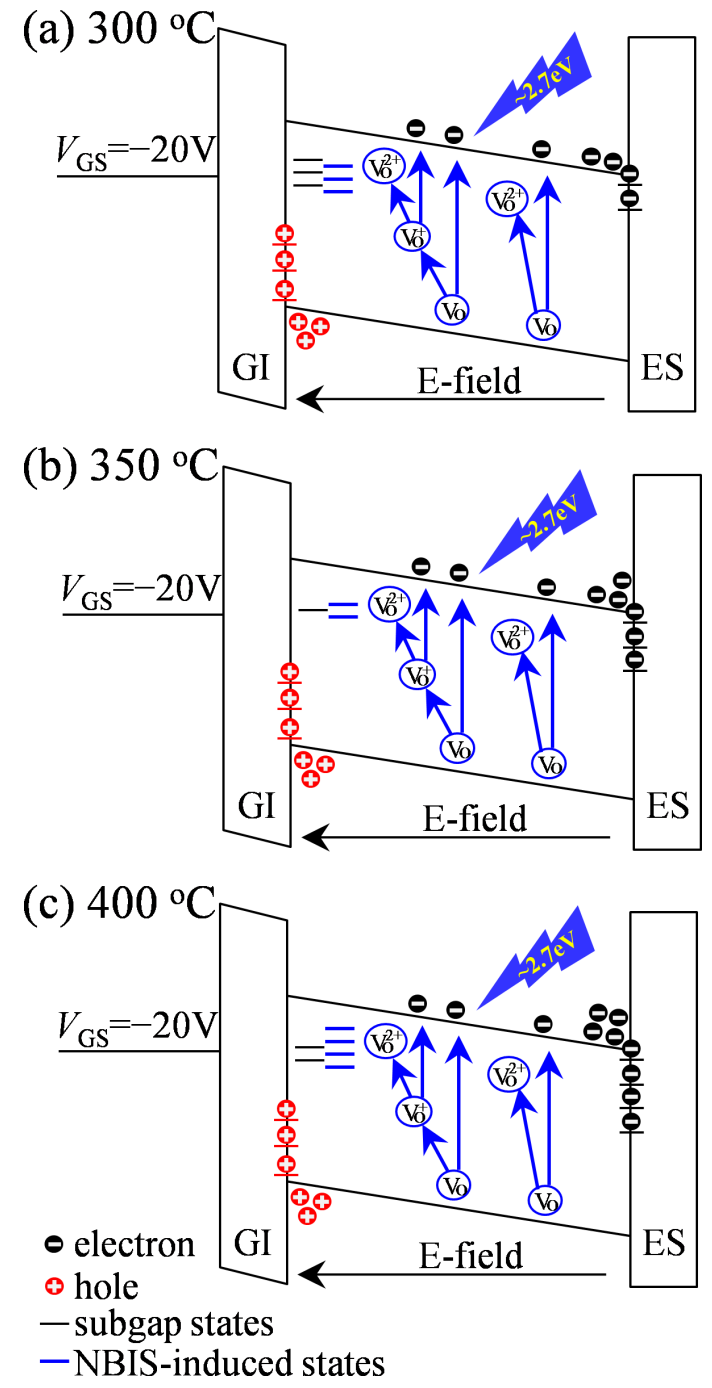

Figure 5: Possible degradation mechanisms of NBIS-induced instability in the devices annealed at (a) 300 , (b) 350 , and (c) $400{ }^{\circ} \mathrm{C}$, respectively.

stress stabilities of the devices. In this study, the TFT annealed at $350{ }^{\circ} \mathrm{C}$ exhibits an outstanding initial performance, but its long-term stability has been deteriorated to some degree under 
the high-energy light irradiation. Therefore, to further improve the electrical properties and bias-stress stabilities, especially against NBIS, the semiconductor active layer with appropriate stoichiometry during the fabrication should be controlled. Also, the interface engineering in high-performance TFTs, including surface treatment and buffer layer introduction $[14,15]$, should be carefully considered.

\section{Conclusion}

The influence of annealing temperature on the initial electrical characteristics and photo-induced instabilities in a-IGZO TFTs is explored. The electrical parameters reveal that annealing at low and high temperatures leads to the creation of high densities of defects in the IGZO bulk and its adjacent interfaces, respectively. The high-energy incident light excites the defect generation and the leakage current increases in the devices regardless of annealing temperature. Using the double-scan mode and a positive gate pulse, the NBIS-caused hysteresis for the TFTs annealed at different temperatures is quantitatively clarified.

\section{Acknowledgements}

The authors acknowledge all support from the National Key Research and Development Program of China (2016YFA0202403), the National Nature Science Foundation of China $(61674098,91733301)$, the Natural Science Foundation of Shaanxi Provincial Department of Education (2017KW023, 2017JM6020), the Fundamental Research Funds for the Central Universities (GK201903052), the Changjiang Scholar and the Innovative Research Team (IRT_14R33), the 111 Project (B14041), and the Chinese National 1000-talent-plan program (Grant No. 111001034). This work was partly supported by JSPS KAKENHI Grant Number 16K06309.

\section{ORCID ${ }^{\circledR}$ iDs}

Dapeng Wang - https://orcid.org/0000-0001-9897-0627

Mamoru Furuta - https://orcid.org/0000-0003-1685-3246

\section{References}

1. Nomura, K.; Ohta, H.; Takagi, A.; Kamiya, T.; Hirano, M.; Hosono, H. Nature 2004, 432, 488-492. doi:10.1038/nature03090

2. Chowdhury, M. D. H.; Um, J. G.; Jang, J. Appl. Phys. Lett. 2014, 105 , 233504. doi:10.1063/1.4903874

3. Shimakawa, S.-i.; Kamada, Y.; Kawaharamura, T.; Wang, D.; Li, C.; Fujita, S.; Hirao, T.; Furuta, M. Jpn. J. Appl. Phys. 2012, 51, 03 CB04. doi:10.7567/jjap.51.03cb04

4. Kimura, M.; Furuta, M.; Kamada, Y.; Hiramatsu, T.; Matsuda, T.; Furuta, H.; Li, C.; Fujita, S.; Hirao, T. IEEE Trans. Electron Devices 2011, 58, 3018-3024. doi:10.1109/ted.2011.2158546

5. Eom, T.-Y.; Ahn, C.-H.; Kang, J.-G.; Salman, M. S.; Lee, S.-Y.; Kim, Y.-H.; Lee, H.-J.; Kang, C.-M.; Kang, C. Appl. Phys. Express 2018, 11, 061104. doi:10.7567/apex.11.061104
6. Kim, J.; Bang, J.; Nakamura, N.; Hosono, H. APL Mater. 2019, 7, 022501. doi:10.1063/1.5053762

7. Lee, M.; Kim, M.; Jo, J.-W.; Park, S. K.; Kim, Y.-H. Appl. Phys. Lett. 2018, 112, 052103. doi:10.1063/1.4999934

8. Wang, D.; Furuta, M. Beilstein J. Nanotechnol. 2018, 9, 2573-2580. doi:10.3762/bjnano.9.239

9. Kim, Y. J.; Yang, B. S.; Oh, S.; Han, S. J.; Lee, H. W.; Heo, J.; Jeong, J. K.; Kim, H. J. ACS Appl. Mater. Interfaces 2013, 5, 3255-3261. doi:10.1021/am400110y

10. Wang, Y.; Sun, X. W.; Goh, G. K. L.; Demir, H. V.; Yu, H. Y. IEEE Trans. Electron Devices 2011, 58, 480-485. doi:10.1109/ted.2010.2091131

11. Wang, D.; Zhao, W.; Furuta, M. J. Phys. D: Appl. Phys. 2019, 52, 235101. doi:10.1088/1361-6463/ab10fc

12. Furuta, M.; Kamada, Y.; Kimura, M.; Hiramatsu, T.; Matsuda, T.; Furuta, H.; Li, C.; Fujita, S.; Hirao, T. IEEE Electron Device Lett. 2010, 31, 1257-1259. doi:10.1109/led.2010.2068276

13. Hung, M. P.; Wang, D.; Jiang, J.; Furuta, M. ECS Solid State Lett. 2014, 3, Q13-Q16. doi:10.1149/2.010403ss

14. Chang, G.-W.; Chang, T.-C.; Jhu, J.-C.; Tsai, T.-M.; Syu, Y.-E.; Chang, K.-C.; Tai, Y.-H.; Jian, F.-Y.; Hung, Y.-C. Appl. Phys. Lett. 2012, 100, 182103. doi:10.1063/1.4709417

15. Kim, S. J.; Kim, H. T.; Choi, J. H.; Chung, H. K.; Cho, S. M. Appl. Phys. Lett. 2018, 112, 152104. doi:10.1063/1.5022714

\section{License and Terms}

This is an Open Access article under the terms of the Creative Commons Attribution License (http://creativecommons.org/licenses/by/4.0). Please note that the reuse, redistribution and reproduction in particular requires that the authors and source are credited.

The license is subject to the Beilstein Journal of

Nanotechnology terms and conditions:

(https://www.beilstein-journals.org/bjnano)

The definitive version of this article is the electronic one which can be found at: doi:10.3762/bjnano.10.112 